\title{
Structural brain assessment of temporal lobe epilepsy based on voxel-based and surface-based morphological features
}

\author{
Zhensheng Li ${ }^{1}$, Jianjie Kang ${ }^{1}$, Quwen Gao ${ }^{1}$, Kairun Peng ${ }^{1}$, Wei Wang ${ }^{2}$, Jian Lin ${ }^{1}$, \\ Weimin $\mathrm{Wang}^{1}$, Xiaofei Guo ${ }^{2}$ \\ ${ }^{1}$ Department of Neurology, General Hospital of Southern Theatre Command, PLA, Guangzhou, China \\ ${ }^{2}$ Department of Neurosurgery, Sun Yat-sen Memorial Hospital, Sun Yat-sen University, Guangzhou, China
}

\section{ABSTRACT}

Aim of the study. This study aimed to assess the cerebral voxel-based and surface-based morphological abnormalities of patients with temporal lobe epilepsy (TLE).

Materials and methods. A total of 100 healthy adults and 73 patients with TLE were enrolled in this study, and their 3D T1-weighted MRI data were collected. Voxel-based morphology (VBM) and surface-based morphology (SBM) tools were used to compare the morphological differences between healthy adults and patients with TLE. Receiver-operating characteristic (ROC) curves were used to acquire the boundary values for detecting morphological abnormalities in regions of interest from the corrected VBM and SBM analysis.

Results. Our results showed that cortical voxels and decreased thickness areas were located in the widespread cortex and subcortical structures in the TLE group. However, after completing the analysis, we found that the left-TLE lesions were limited to the left temporal pole and left hippocampus, while the right-TLE lesions were located in the bilateral medial temporal lobe, including the right hippocampus and bilateral amygdala. ROC-curve results showed that the volume of the left hippocampus at $4,124.45 \mathrm{~mm}^{3}$ and the thickness of the left temporal pole cortex at $3.50 \mathrm{~mm}$ could be used as optimal boundary values based on the curves of the left-TLE group. The right-TLE group curves were poor.

Conclusions. Widespread cerebral morphological TLE abnormalities were represented in this study. However, the lesions may be limited after completing a corrected comparison with clinical information. Boundary values of left-TLE group lesions were also obtained. Key words: structural assessment, temporal lobe epilepsy, morphological atlas, hippocampal sclerosis, 3D T1-weighted images (Neurol Neurochir Pol 2021; 55 (4): 369-379)

\section{Introduction}

Temporal lobe epilepsy (TLE) is a common type of drug-resistant epilepsy [1]. Research studies have shown that patients with TLE suffer from widespread cortical and subcortical morphological abnormalities, such as decreased volume and cortical atrophy. The regions were mostly located in the frontal, temporal, occipital, and central cortex, and the thalamus $[2,3]$. These studies have also found damage in white matter fibres [4]. Therefore, it is expected that patients with TLE suffer a certain degree of cognitive impairment, in addition to frequent seizures, that are closely related to the structural abnormalities $[5,6]$. A certain amount of neural network damage is also related to cerebral emotional functions [7]. It is considered that factors such as long-term duration, frequent seizures, and medication have an influence on the impairments of the cortical and subcortical structures [8], but it has also been found that progressive cortical atrophy is still present with the disease's prolongation, even in the case of seizure freedom [9]. It is currently believed that epilepsy is a neurodegenerative disease, and the spread of epileptiform discharges are considered to be detrimental to the widespread cortical and subcortical structures [10].

Address for correspondence: Weimin Wang, Department of Neurosurgery, General Hospital of Southern Theatre Command, PLA, No. 111 of Liuhua Road, Yuexiu District, Guangzhou, 510010, China; e-mail: weimin_wang11@163.com

Received: 20.02.2021 Accepted: 27.04.2021 Early publication date: 2.06.2021 
Quantitative measurements have been used as a non-invasive tool for brain morphology assessments, and the effects have also been shown to be consistent with actual measurements [11]. However, widespread cortical atrophy has been discovered in patients with TLE, with no benefits in terms of locating the epileptogenic lesions. Fully quantitative cerebral morphological assessments could provide a path for predicting curative effects, cognitive evaluation, and early surgical decisions [12]. They also provide precise boundary evaluations for detecting morphological abnormalities that have been difficult to recognise in conventional imaging. Therefore, this study aimed to assess the cerebral morphology for patients with TLE based on voxel-based morphometry (VBM) [13] and surface-based morphometry (SBM) [14].

\section{Materials and methods}

\section{Subjects}

In this study, we collected the 3D T1-weighted MRI data of healthy adults and patients with TLE who were recruited from 2015 to 2019.

Standard protocol approvals, registration, and participant consent: all participants provided written consent. The institutional review board at the General Hospital of Southern Theatre Command, PLA, approved this study.

\section{Inclusion and exclusion criteria}

In the healthy adults' group, the inclusion criteria were: 1. healthy adult; 2 . aged over $18 ; 3$. right-handed; 4 . EEG and cerebral MR examinations taken with normal results. The exclusion criteria were : 1 . a history of neurological or mental illness; 2. a history of chronic disease such as hypertension, diabetes, or coronary heart disease; 3 . a positive family history of hereditary disease.

In the TLE group, the inclusion criteria were: 1. patients with unilateral TLE, with a diagnosis that fulfilled the criteria of the International League Against Epilepsy (ILAE) [15] (this criterion was established based on a comprehensive investigation that included the collection of seizure semiology, the inspection of discharge from unilateral temporal areas on inter-ictal/ictal EEGs, video-telemetry recordings, and the inspection of MR images for evidence of structural abnormalities in the mesial temporal lobe); 2. Aged over 16. The exclusion criteria were: 1 . Patients with TLE caused by inflammation; 2. Patients with TLE caused by trauma; 3 . Patients with TLE caused by brain tumors; 4 . Patients with bilateral TLE.

\section{Materials}

General demographic information, such as the gender and age of each participant, was collected. For patients with TLE, it was also necessary to collect clinical information such as disease duration, age at onset, seizure types, seizure frequency, and medication use (Tab. 1).
MR acquisition: The visual interpretation of the findings in this study was conducted by an experienced clinician. Cerebral MR scans were performed on 173 participants using a General Electric (GE) 3.0T MR scanner and a SIEMENS 3.0T MR scanner. The scan sequences included the conventional T1-weighted, T2-weighted, and T2 FLAIR images. Three-dimensional T1-weighted MPRAGE sequences $(\mathrm{TE}=3.24 \mathrm{~ms}$, $\mathrm{TR}=2300 \mathrm{~ms}, \mathrm{TI}=900 \mathrm{~ms}$, flip angle $=9$ degrees, bandwidth $=210 \mathrm{~Hz} /$ pixel, FOV $=256 \mathrm{~mm}$, matrix $=256 \times 256$, resolution $1.0 \times 1.0 \times 1.0 \mathrm{~mm}^{3}$, a total of 176 sagittal images) were also necessary.

\section{Methods}

SPM [16] was used for the cortical assessment of TLE patients.

For the 3D T1-weighted magnetisation, prepared rapid gradient echo (MPRAGE) sequences were preprocessed, including the DARTEL normalisation of images to MNI152 spacing, the segmentation of grey matter (GM), white matter (WM) and cerebrospinal fluid (CSF), and the estimation of cortical surface and thickness and smooth areas.

Before entering the GM images into a statistical model, GM-image data was required for the estimation of the cortical surface and thickness and the smooth areas through the 'Estimate Surface Models' function.

The cortical surface images and smoothed GM images were then entered into the statistical models for a comparison between the healthy group and the group with TLE.

The subcortical structures, including the hippocampus, amygdala, caudate nucleus, putamen, globus pallidus, nucleus accumbens, and the brainstem, were segmented and reconstructed within a Freesurfer 5.3 software package [17]. The T1-weighted NIFTI format images were input into Freesurfer and automatically reconstructed through the 'reconall' function. These processes included removing non-brain tissue, volumetric labelling, intensity normalisation, GM and WM segmentation, subcortical mass creation, and data estimation (Fig. 1).

\section{Statistical analysis}

T-test statistical models using the standard VBM and SBM 'Specify 2nd Level' or 'Basic Models' modules were performed for grey-matter volume and thickness comparison at two different significance thresholds: an uncorrected threshold of $\mathrm{p}<0.001$ and a threshold of $\mathrm{p}<0.05$ corrected for FDR. Comparisons were performed again when corrected by covariates, including disease duration, age at onset, seizure type, seizure frequency, number of current medications, and total intracranial volume. The regions of interest (ROIs) were then detected from these comparisons. SPSS 19.0 software program (IBM, Chicago, IL, USA) was used for statistical data. The cortical thickness data of ROIs were derived from SBM analysis, 

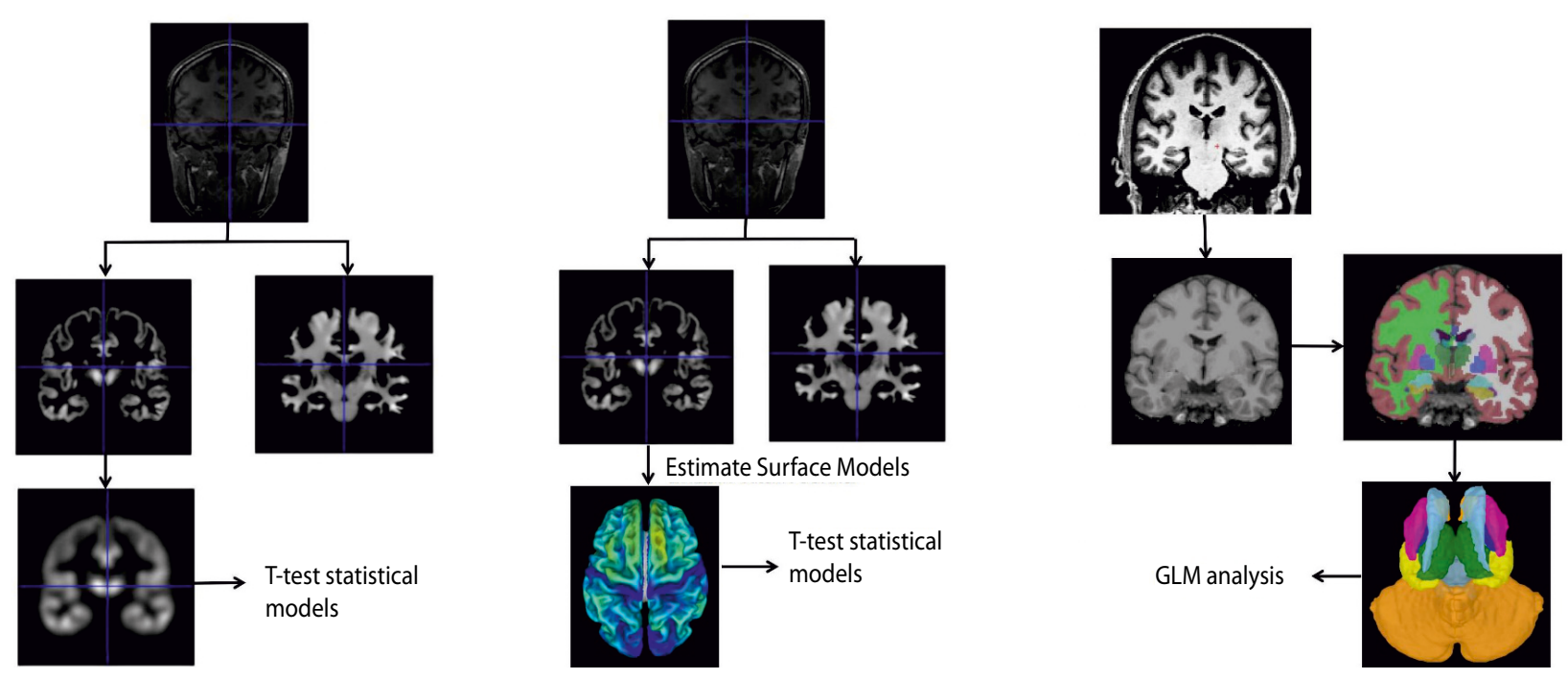

Figure 1. Processing streams for 3D T1-weighted magnetisation: prepared rapid gradient echo (MPRAGE) sequences

and the subcortical structural volumes were derived from the Freesurfer 5.3 results. The data was imported into the SPSS. A general linear model (GLM) was used for the covariance comparison of cortical and subcortical structural data between the groups. We used the covariates above for correction and the ROC curves to acquire the boundary values with the highest sensitivity and specificity for detecting morphological abnormalities. A value of $\mathrm{p}<0.05$ was considered statistically significant.

\section{Results}

\section{General characteristics}

We recruited 100 healthy adults for the first group. These included 50 males and 50 females, and their ages ranged from 18 to 40 . We recruited 73 patients with TLE for the second group, including 38 left-TLE patients and 35 right-TLE patients. Their ages ranged from 16 to 44 .

\section{Cortical morphological comparison between healthy group and TLE group}

The VBM comparison results between the groups showed that voxel-decreased areas in patients with TLE were found in the widespread cortical and subcortical structures in addition to the temporal lobe, including the bilateral cingulate, temporal cortex, and thalamus ( $\mathrm{p}<0.001$, uncorrected). The cortical thickness comparison results based on the SBM analysis between the groups were shown based on the Destrieux 2009 parcellation [18]. In addition, we found that the cortical thickness atrophy areas of the patients with TLE were also present in the widespread cortex, with the most obvious areas in the bilateral frontal, temporal, and central cortex (Fig. 2, 3) ( $\mathrm{p}<0.05$, FDR corrected). The cortical atrophy ranges of the left TLE were more extensive than for the right TLE.

\section{Corrected cortical morphological comparison} between healthy and TLE groups

The corrected VBM comparisons between the healthy group and the left-TLE patients demonstrated that the morphologically abnormal areas were limited to the left hippocampus ( $\mathrm{p}<0.001$, uncorrected), while the morphologically abnormal areas in the patients with right TLE were found in the bilateral medial temporal lobe, based on the corrected VBM comparisons. In the corrected SBM analysis, the cortical atrophy area of the patients with left TLE was confined to the left temporal pole area (original data $3.71 \pm 0.27 \mathrm{~mm} v s .3 .48$ $\pm 0.45 \mathrm{~mm}$, adjusted $\mathrm{p}=0.027$ ). No significant results were found in the corrected SBM analysis between the healthy group and the right-TLE group (Fig. 4, 5).

\section{Corrected subcortical structure volume} comparison between healthy and TLE groups

The corrected comparison of the subcortical structure volumes by GLM also confirmed that the left hippocampus volume of the left-TLE patients was reduced (original data $4,325.62 \pm 454.70 \mathrm{~mm}^{3}$ vs. $3,849.32 \pm 761.36 \mathrm{~mm}^{3}$, adjusted $\mathrm{p}=0.033)$. The volume-decreased areas were located in the right hippocampus $\left(4,542.17 \pm 418.28 \mathrm{~mm}^{3} v\right.$ s. $4,049.94$ $\pm 985.20 \mathrm{~mm}^{3}$, adjusted $\mathrm{p}=0.002$ ) and the bilateral amygdala (left side: $1,685.50 \pm 214.54 \mathrm{~mm}^{3} v s .1,504.43 \pm 227.38 \mathrm{~mm}^{3}$, adjusted $\mathrm{p}=0.003$, right side: $1,717.50 \pm 170.92 \mathrm{~mm}^{3} v s .1,563.72$ $\pm 269.38 \mathrm{~mm}^{3}$, adjusted $\mathrm{p}=0.001$ ) of the right-TLE patients.

\section{ROC curves for detection of morphological abnormalities in ROIs}

For the left-TLE patients, ROC curves of the left hippocampus volume and left temporal pole area thickness were performed. The area under the ROC curve of the left hippocampus volume was 0.699 , and the optimal boundary value 
Table 1. Demographic characteristics and clinical information of healthy adults and TLE patients

\begin{tabular}{|c|c|c|c|c|c|c|}
\hline Index & & $\begin{array}{c}\text { Healthy } \\
\text { adults }\end{array}$ & Left-TLE group & Right-TLE group & $F / X^{2}$ & $\mathbf{P}$ \\
\hline Age (years) & & $27.71 \pm 4.00$ & $26.84 \pm 8.52$ & $26.86 \pm 8.34$ & 0.401 & 0.670 \\
\hline \multirow[t]{2}{*}{ Sex } & Male & $50(50)$ & 25 (65.8\%) & 15 (42.9\%) & \multirow[t]{2}{*}{4.227} & \multirow[t]{2}{*}{0.121} \\
\hline & Female & $50(50)$ & $13(34.2 \%)$ & $20(57.1 \%)$ & & \\
\hline \multirow{4}{*}{$\begin{array}{l}\text { Disease duration } \\
\text { (years) }\end{array}$} & $0-5$ & - & $16(42.1 \%)$ & $14(40.0 \%)$ & \multirow[t]{4}{*}{1.732} & \multirow[t]{4}{*}{0.630} \\
\hline & $6-10$ & - & $9(23.7 \%)$ & $10(28.6 \%)$ & & \\
\hline & $11-15$ & - & $8(21.1 \%)$ & $4(11.4 \%)$ & & \\
\hline & $\geq 16$ & - & $5(13.2 \%)$ & $7(20.0 \%)$ & & \\
\hline \multirow[t]{4}{*}{ Onset age (years) } & $0-10$ & - & $6(15.8 \%)$ & $7(20.0 \%)$ & \multirow[t]{4}{*}{0.302} & \multirow[t]{4}{*}{0.960} \\
\hline & $11-20$ & - & $19(50.0 \%)$ & $16(45.7 \%)$ & & \\
\hline & $21-30$ & - & $7(18.4 \%)$ & $7(20.0 \%)$ & & \\
\hline & $\geq 16$ & - & $6(15.8 \%)$ & $5(14.3 \%)$ & & \\
\hline \multirow[t]{2}{*}{ Seizure type } & CPS & - & $28(73.7 \%)$ & $23(65.7 \%)$ & 0.55 & 0.458 \\
\hline & sGTCS & - & $34(89.5 \%)$ & 31 (88.6\%) & 0.015 & 0.902 \\
\hline \multirow{5}{*}{$\begin{array}{l}\text { CPS frequency } \\
\text { (times per year) }\end{array}$} & $<10$ & - & $3(7.9 \%)$ & $6(17.6 \%)$ & \multirow[t]{5}{*}{5.966} & \multirow[t]{5}{*}{0.31} \\
\hline & $10-20$ & - & $6(15.8 \%)$ & $4(11.4 \%)$ & & \\
\hline & $21-30$ & - & $5(13.2 \%)$ & $3(8.6 \%)$ & & \\
\hline & $31-40$ & - & $4(10.5 \%)$ & $0(0 \%)$ & & \\
\hline & $>41$ & - & $9(23.7 \%)$ & $9(25.7 \%)$ & & \\
\hline \multirow{5}{*}{$\begin{array}{l}\text { sGTCS frequency } \\
\text { (times per year) }\end{array}$} & $<10$ & - & $17(44.7 \%)$ & 22 (62.9\%) & \multirow[t]{5}{*}{7.109} & \multirow[t]{5}{*}{0.213} \\
\hline & $10-20$ & - & $13(34.2 \%)$ & $6(17.1 \%)$ & & \\
\hline & $21-30$ & - & $0(0 \%)$ & $2(5.7 \%)$ & & \\
\hline & $31-40$ & - & $1(2.6 \%)$ & $0(0 \%)$ & & \\
\hline & $>41$ & - & $3(7.9 \%)$ & $1(2.9 \%)$ & & \\
\hline Febrile seizures history & & - & $13(34.20 \%)$ & $7(20.0 \%)$ & 1.85 & 0.174 \\
\hline Family history & & - & $1(2.6 \%)$ & $1(2.9 \%)$ & 0.003 & 0.953 \\
\hline \multirow[t]{8}{*}{ Medication } & Sodium Valproate & - & $16(42.1 \%)$ & $19(54.3 \%)$ & 1.083 & 0.298 \\
\hline & Carbamazepine & - & $11(28.9 \%)$ & $7(20 \%)$ & 0.785 & 0.376 \\
\hline & Oxcarbazepine & - & $4(10.5 \%)$ & $3(8.6 \%)$ & 0.08 & 0.777 \\
\hline & Topiramate & - & $5(13.2 \%)$ & $4(11.4 \%)$ & 0.05 & 0.822 \\
\hline & Lamotrigine & - & $1(2.6 \%)$ & $10(28.6)$ & 9.579 & 0.02 \\
\hline & Levetiracetam & - & $3(7.9 \%)$ & $4(11.4 \%)$ & 0.262 & 0.608 \\
\hline & Phenytoin & - & $2(5.3 \%)$ & $1(2.9 \%)$ & 0.268 & 0.605 \\
\hline & Benzodiazepine & - & $4(10.5 \%)$ & $2(5.7 \%)$ & 0.559 & 0.455 \\
\hline
\end{tabular}

CPS - complex partial seizures; sGTCS - secondary generalised tonic-clonic seizures

was $4,124.45 \mathrm{~mm}^{3}$, with sensitivity $60.5 \%$ and specificity $77.0 \%$. The area under the ROC curve of the left temporal pole thickness was 0.711 , and the optimal boundary value was $3.50 \mathrm{~mm}$, with sensitivity $60.5 \%$ and specificity $78.0 \%$. However, for the right-TLE patients, the ROC curves for the right hippocampus and amygdala volume showed poor efficiency. The area under the ROC curve of the right hippocampus volume was 0.605 , and the optimal boundary value was $4,487.95 \mathrm{~mm}^{3}$, with sensitivity only $57.1 \%$ and specificity $58.0 \%$. The area under the ROC curve of the right amygdala volume was 0.679 , and the optimal boundary value was $1,658.45 \mathrm{~mm}^{3}$, with sensitivity $62.9 \%$ and specificity $64.0 \%$ (Fig. 6).

\section{Discussion}

This study showed that grey-matter volume and cortical thickness-decreased areas were located in the widespread cortex and subcortical structures in the TLE group. However, after our corrected analysis, we found that left-TLE lesions were limited to the left temporal pole and left hippocampus. 


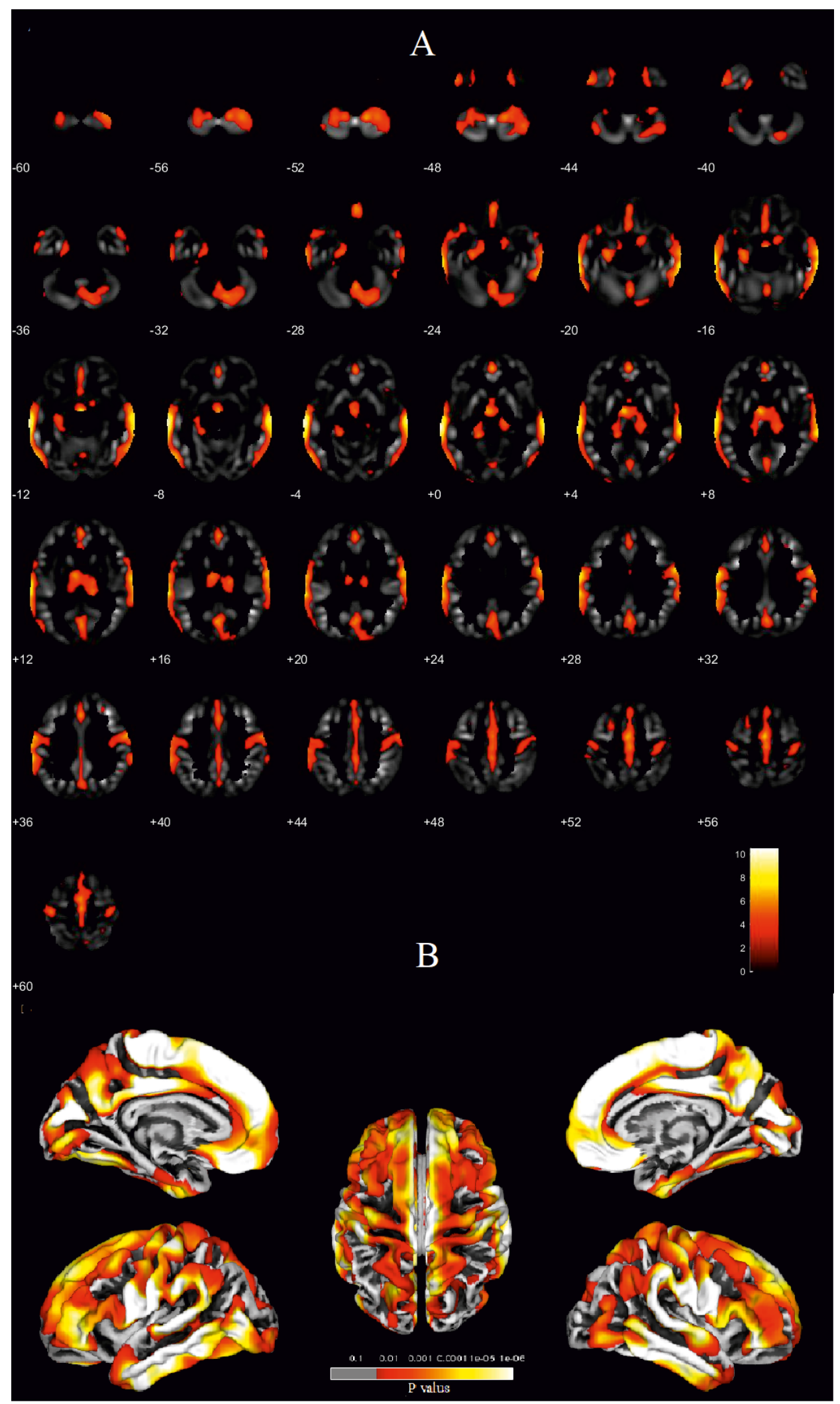

Figure 2. VBM and SBM analysis between healthy group and left-TLE patients 


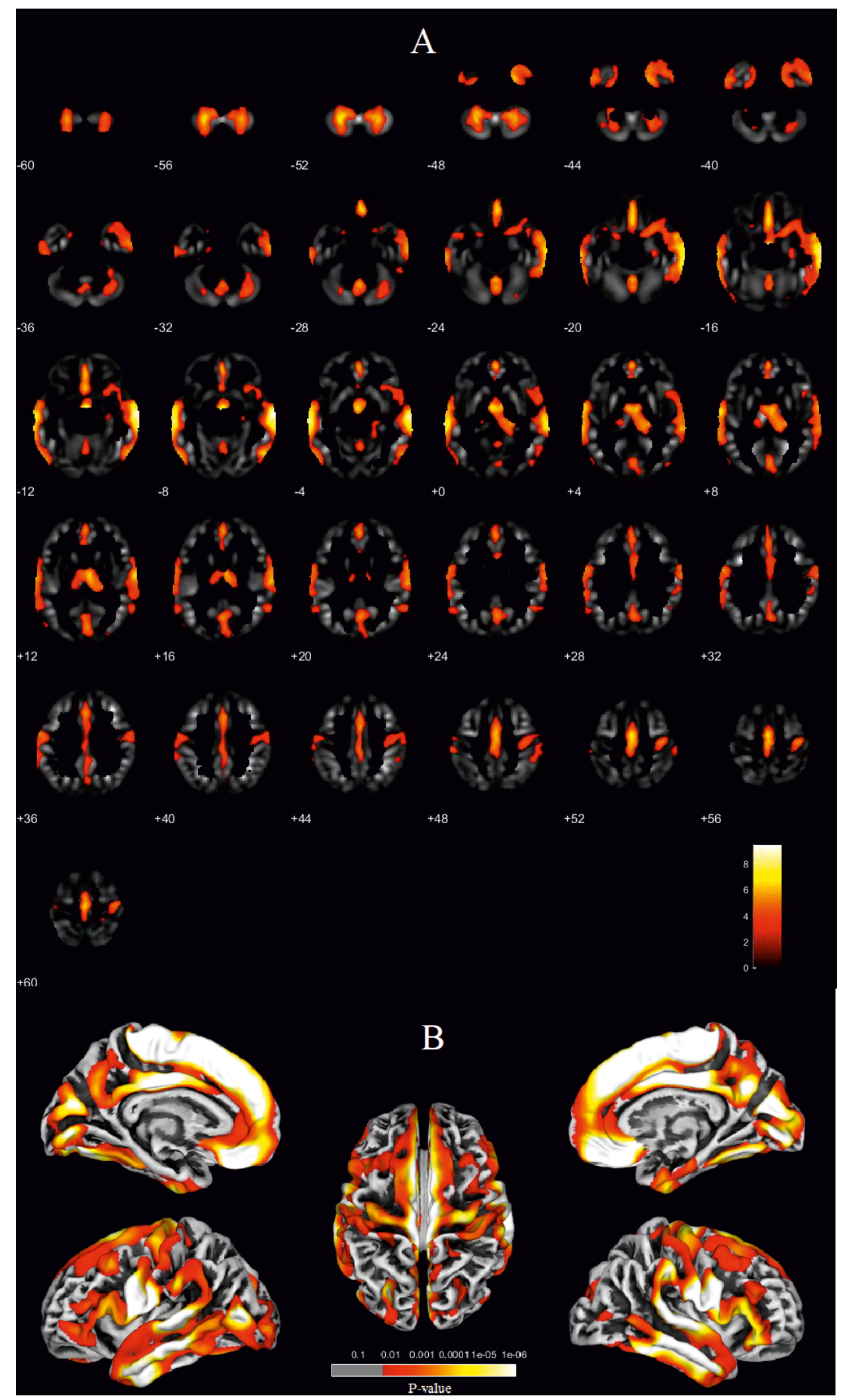

Figure 3. VBM and SBM analysis between healthy group and right-TLE patients 


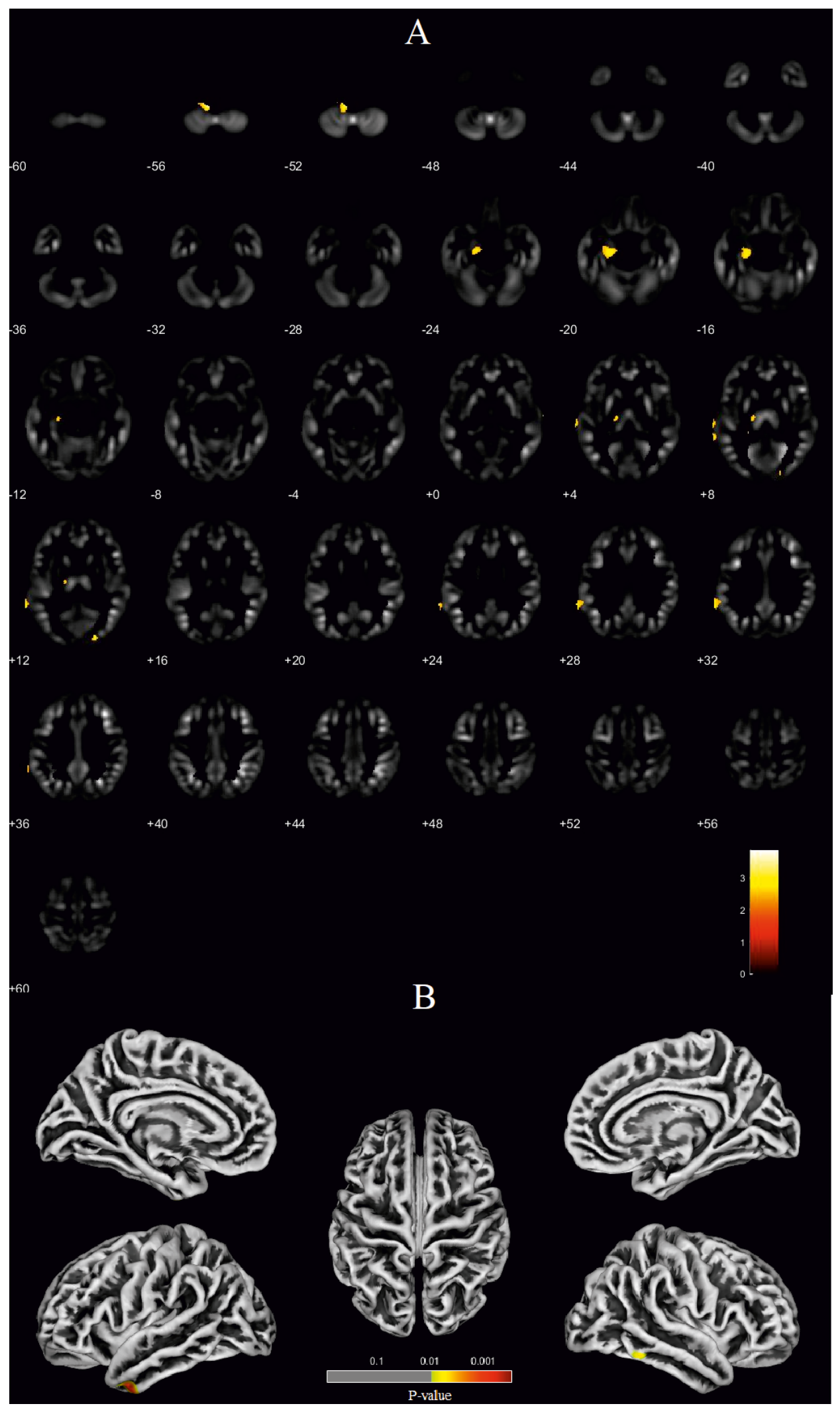

Figure 4. Corrected VBM and SBM analysis between healthy and left-TLE groups 


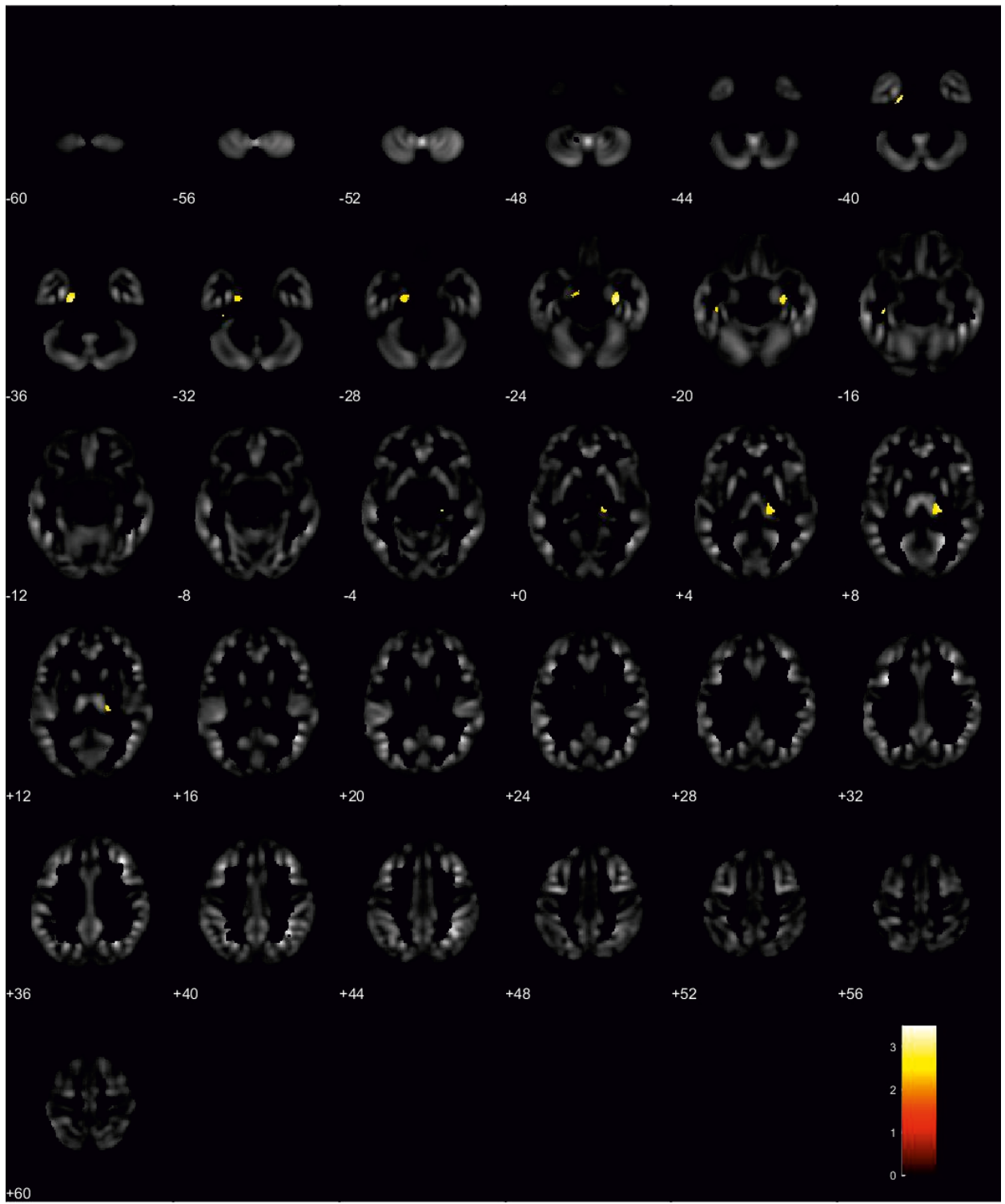

Figure 5. Corrected VBM analysis between healthy and right-TLE groups

The lesions in patients with right TLE were located in the bilateral medial temporal lobe, including the right hippocampus and bilateral amygdala. The results of the ROC curves showed that the volume of the left hippocampus at $4,124.45 \mathrm{~mm}^{3}$ and the thickness of the left temporal pole cortex at $3.50 \mathrm{~mm}$ could be used as the optimal boundary values based on the left-TLE group curves. However, the right-TLE results were relatively insignificant.

Based on innovations in imaging processing methods, especially the widespread application of VBM, it has been reported that temporal lobe epilepsy is a network disorder. Even a multicentric study of advanced morphological neuroimaging in lateral temporal lobe epilepsy has been performed in recent years [19].

We found similar results in the uncorrected VBM and SBM analysis in this study, demonstrating widespread cortical and sub-cortical atrophy areas in TLE. However, these results have no benefits for lesion localisation, something which is also highlighted in previous studies. VBM based on grey-matter volume appeared unfavourable in presurgical focal epilepsy patients, but VBM based on T2-FLAIR and junction maps might achieve higher specificity and sensitivity [20].

Furthermore, as discussed above, these results were mixed, with many confounding clinical factors. Some scholars have been able to detect morphological alterations confined to the medial temporal lobe after excluding the influence of disease duration and onset age [21]. But these studies were only focused on hippocampal sclerosis patients, which is not conducive to determining morphological abnormalities in patients who have normal imaging on visual inspection. Nor was medication involved in the cerebral 


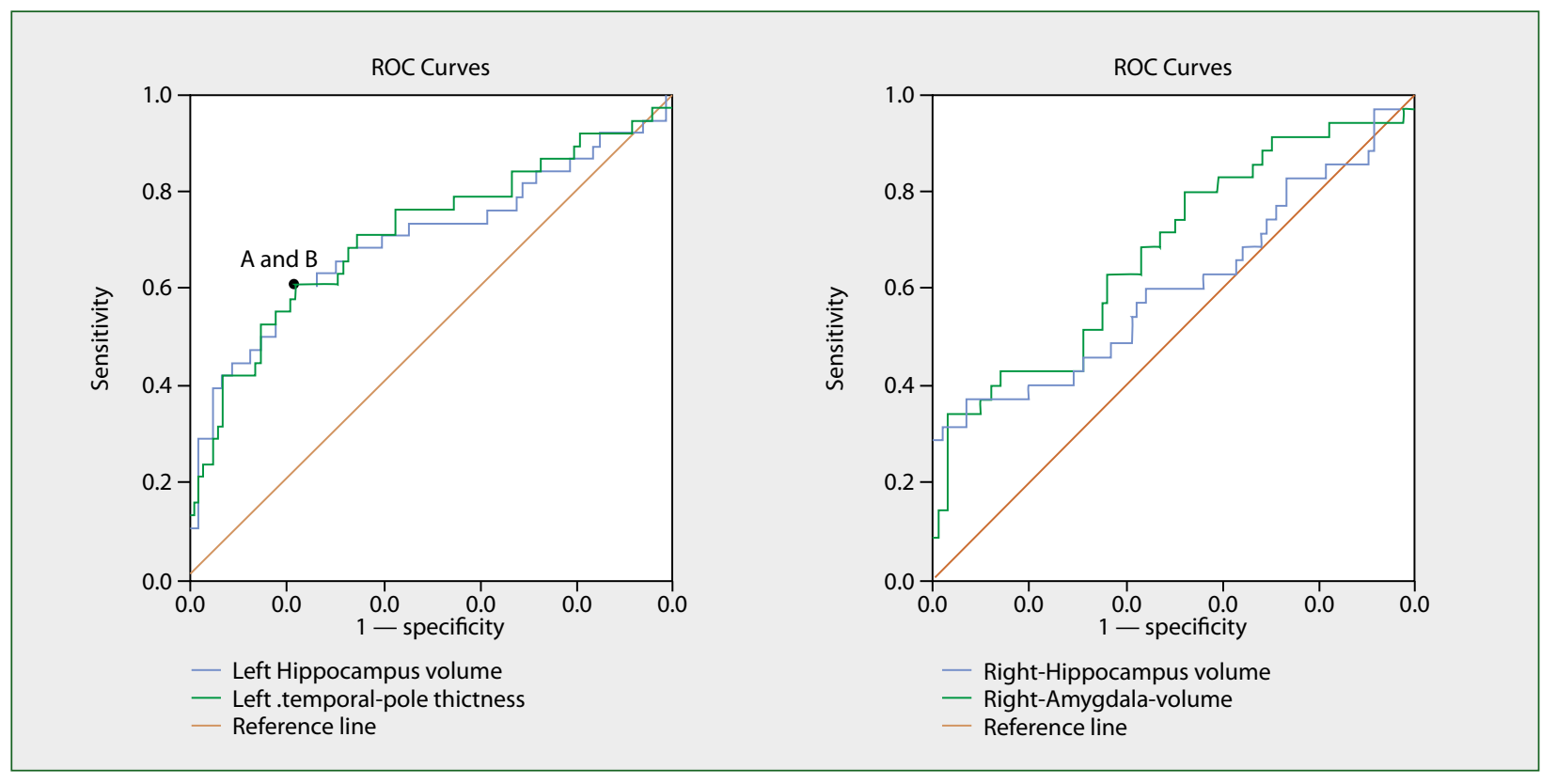

Figure 6. ROC curves of ROls for detecting morphological abnormalities in patients with TLE

morphological changes of the patients with TLE. For this reason, we recruited TLE patients who even had MRI negative conditions, and we also considered the influence of medication factors.

A decrease in the left hippocampus volume and left temporal pole cortical thickness was the main morphological alteration in the left-TLE patients, who still had to be identified by corrected VBM and SBM analysis, respectively, in this study. The volume decrease of the left hippocampus was also supported by a corrected comparison of subcortical structure volumes. The volume decrease in the TLE hippocampus has long been documented and is related to memory impairment [22]. Moreover, studies concerning the cortical thickness of the left-TLE lesions showed the left temporal polar thickness thinning specifically. This result could provide evidence for the early detection of temporal cortical dysplasia. Likewise, the most significant morphological change in right-TLE patients was right hippocampus volume loss.

Thus, it can be concluded that ipsilateral hippocampus volume decrease was still the most intrinsic morphological abnormality of unilateral TLE. Quantitative morphological analysis for TLE has been refined in hippocampal subfields [23]. The CA1 field is receiving attention from researchers and has proved to be related to the histopathology in TLE [24]; however, total hippocampal volume atrophy was found in the patients of this study, suggesting that hippocampal subfields other than CA1 should be considered equally in the morphological assessment of TLE. We expect to prove this assumption in further studies. In addition, the results also suggest that confounding factors had a considerable influence on extensive extratemporal cortical damage. These factors need to be excluded in lesion localisation, especially in the pre-surgery assessment.

We further attempted to use ROC curves to analyse the ROI data from the corrected VBM and SBM analysis to determine the criteria of morphological abnormalities for left-TLE patients. This showed that the areas under the ROC curves of the left hippocampus and left temporal pole reached 0.7 . The volume of the left hippocampus at $4,124.45 \mathrm{~mm}^{3}$ and the cortical thickness of the left temporal pole at $3.50 \mathrm{~mm}$ was the optimal boundary value based on the current curves. However, the results of the ROC curves of the right-TLE group were quite poor.

Under the corrected comparison, the grey-matter volume decrease in the bilateral medial temporal lobe was found in the corrected VBM analysis of the right-TLE patients. The corrected GLM analysis of the subcortical structures also confirmed a volume decrease in the right hippocampus and bilateral amygdala areas. These results are in contrast to studies that have suggested more widespread abnormalities with left TLE than right TLE $[2,3]$. In fact, the results of our uncorrected morphological analysis support previous conclusions. The different results between the left and right TLE in the corrected analysis may suggest a variance in the intrinsic epileptic networks in patients with unilateral TLE. Another recent study has also confirmed a contralateral shape inflation of the left hippocampus in the right TLE [25]. The connectivity between bilateral temporal lobes has been clearly shown [26, 27]. Furthermore, another study using interictal connectivity analysis on a high-density EEG found a stronger connection from the ipsilateral to the contralateral medial temporal pole in the right TLE than the left TLE [28]. 
It could be assumed that the seizures in right-TLE patients are more likely to be caused by a linkage between bilateral medial temporal lobe structures. This also suggests that we should pay more attention to the possibility of contralateral temporal lobe damage in the clinical assessment of patients with right TLE.

There were several limitations to this study. Firstly, this study was a single-centre trial, and the sample size was limited. Secondly, the ROC-curve analysis failed to achieve higher sensitivity and specificity, which may be due to the use of raw data. An accurate correction algorithm is required to obtain corrections for better boundaries. Thirdly, we only used VBM and SBM tools to validate morphological abnormalities. More structural and functional data, such as diffusion tensor imaging (DTI), EEG, and fMRI, should be included in future clinical assessments. Fourthly, in this study, hippocampal volumes and volumetric measurements were not corrected for total intracranial volume, which may have caused a certain bias. Finally, no pathological information was provided in this study. This should be included in future studies.

\section{Conclusions}

Widespread cerebral morphological TLE abnormalities were presented in this study. However, the lesions could be limited after a corrected comparison with the clinical information. The boundary values of the left-TLE group lesions were also obtained.

Acknowledgments: The authors thank the patients and healthy adults who took part in this study.

Funding: This work was supported by the National Technology Research and Development Programme (863 programme) of China (2015137). The funding body had no role in the design of the study and collection, analysis and interpretation of data, or in writing the manuscript.

Conflicts of interest: None.

Ethical permission: This study was approved by the institutional review board at the General Hospital of Southern Theatre Command, PLA.

\section{References}

1. Sanz-Garcia A, Vega-Zelaya L, Pastor J, et al. When does Post-Ictal period start in temporal lobe epilepsy? A quantitative EEG perspective. Rev Neurol. 2017; 64(8): 337-346, indexed in Pubmed: 28368080.

2. Whelan CD, Altmann A, Botía JA, et al. Structural brain abnormalities in the common epilepsies assessed in a worldwide ENIGMA study. Brain. 2018; 141(2): 391-408, doi: 10.1093/brain/awx341, indexed in Pubmed: 29365066.

3. Bernasconi N, Bernhardt B, Concha L, et al. Cortical thickness analysis in temporal lobe epilepsy: reproducibility and relation to outcome. Neurology. 2010; 74(22): 1776-1784, doi: 10.1212/ WNL.0b013e3181e0f80a, indexed in Pubmed: 20513813.
4. Xu Y, Qiu S, Wang J, et al. Disrupted topological properties of brain white matter networks in left temporal lobe epilepsy: a diffusion tensor imaging study. Neuroscience. 2014; 279: 155-167, doi: 10.1016/j. neuroscience.2014.08.040, indexed in Pubmed: 25194789.

5. Bell B, Lin JJ, Seidenberg M, et al. The neurobiology of cognitive disorders in temporal lobe epilepsy. Nat Rev Neurol. 2011; 7(3): 154-164, doi: 10.1038/nrneurol.2011.3, indexed in Pubmed: 21304484.

6. Keller SS, Richardson MP, O'Muircheartaigh J, et al. Morphometric MRI alterations and postoperative seizure control in refractory temporal lobe epilepsy. Hum Brain Mapp. 2015; 36(5): 1637-1647, doi: 10.1002/hbm.22722, indexed in Pubmed: 25704244.

7. Toller G, Adhimoolam B, Rankin KP, et al. Right fronto-limbic atrophy is associated with reduced empathy in refractory unilateral mesial temporal lobe epilepsy. Neuropsychologia. 2015; 78: 80-87, doi: 10.1016/j.neuropsychologia.2015.09.010, indexed in Pubmed: 26363299.

8. Caciagli L, Bernasconi A, Wiebe S, et al. A meta-analysis on progressive atrophy in intractable temporal lobe epilepsy: Time is brain? Neurology. 2017; 89(5): 506-516, doi: 10.1212/WNL.0000000000004176, indexed in Pubmed: 28687722.

9. Alvim M, Coan A, Campos B, et al. Progression of gray matter atrophy in seizure-free patients with temporal lobe epilepsy. Epilepsia. 2016; 57(4): 621-629, doi: 10.1111/epi.13334.

10. Bernasconi $\mathrm{N}$. Is epilepsy a curable neurodegenerative disease? Brain. 2016; 139(Pt 9): 2336-2337, doi: 10.1093/brain/aww202, indexed in Pubmed: 27559105.

11. Fischl B, Dale AM. Measuring the thickness of the human cerebral cortex from magnetic resonance images. Proc Natl Acad Sci U S A. 2000; 97(20): 11050-11055, doi: 10.1073/pnas.200033797, indexed in Pubmed: 10984517.

12. He X, Doucet GE, Pustina D, et al. Presurgical thalamic "hubness" predicts surgical outcome in temporal lobe epilepsy. Neurology. 2017; 88(24): 2285-2293, doi: 10.1212/WNL.00000000000004035, indexed in Pubmed: 28515267.

13. Good CD, Johnsrude IS, Ashburner J, et al. A voxel-based morphometric study of ageing in 465 normal adult human brains. Neuroimage. 2001; 14(1 Pt 1): 21-36, doi: 10.1006/nimg.2001.0786, indexed in Pubmed: 11525331.

14. Dahnke R, Yotter RA, Gaser C. Cortical thickness and central surface estimation. Neuroimage. 2013; 65: 336-348, doi: 10.1016/j.neuroimage.2012.09.050, indexed in Pubmed: 23041529.

15. Berg AT, Berkovic SF, Brodie MJ, et al. Revised terminology and concepts for organization of seizures and epilepsies: report of the ILAE Commission on Classification and Terminology, 2005-2009. Epilepsia. 2010; 51(4): 676-85, doi: 10.1111/j.1528-1167.2010.02522.x, indexed in Pubmed: 20196795.

16. Gaser C, Dahnke RC. A Computational Anatomy Toolbox for the Analysis of Structural MRI Data. HBM. : 2016.

17. Fischl B. FreeSurfer. Neurolmage. 2012; 62(2): 774-781, doi: 10.1016/j.neuroimage.2012.01.021.

18. Fischl B, van de, Destrieux C. Automatically Parcellating the Human. Cerebral Cortex. 2004; 14(1): 11-22, doi: https://doi.org/10.1093/ cercor/bhg087.

19. Vasta R, Sarica A, Bisulli F, et al. Advanced morphological neuroimaging study in lateral temporal lobe epilepsy: A multicentric study. Epilepsy \& Behavior. 2017; 74: 69-72, doi: 10.1016/j.yebeh.2017.06.017. 
20. Martin P, Winston GP, Bartlett P, et al. Voxel-based magnetic resonance image postprocessing in epilepsy. Epilepsia. 2017; 58(9): 1653-1664, doi: 10.1111/epi.13851, indexed in Pubmed: 28745400.

21. Alhusaini S, Whelan CD, Doherty CP, et al. Temporal cortex morphology in mesial temporal lobe epilepsy patients and their asymptomatic siblings. Cereb Cortex. 2016; 26(3): 1234-1241, doi: 10.1093/cercor/bhu315, indexed in Pubmed: 25576532.

22. Bernhardt BC, Worsley $\mathrm{KJ}$, Kim H, et al. Longitudinal and cross-sectional analysis of atrophy in pharmacoresistant temporal lobe epilepsy. Neurology. 2009; 72(20): 1747-1754, doi: 10.1212/01.wnl.0000345969.57574.f5, indexed in Pubmed: 19246420.

23. Bernhardt BC, Fadaie F, Vos de Wael R, et al. Preferential susceptibility of limbic cortices to microstructural damage in temporal lobe epilepsy: A quantitative T1 mapping study. Neuroimage. 2018; 182: 294303, doi: 10.1016/j.neuroimage.2017.06.002, indexed in Pubmed: 28583883.

24. Schoene-Bake JC, Keller SS, Niehusmann P, et al. In vivo mapping of hippocampal subfields in mesial temporal lobe epilepsy: relation to histopathology. Hum Brain Mapp. 2014; 35(9): 4718-4728, doi: 10.1002/hbm.22506, indexed in Pubmed: 24638919.

25. Yoo JG, Jakabek D, Ljung $H$, et al. MRI morphology of the hippocampus in drug-resistant temporal lobe epilepsy: Shape inflation of left hippocampus and correlation of right-sided hippocampal volume and shape with visuospatial function in patients with right-sided TLE. J Clin Neurosci. 2019; 67: 68-74, doi: 10.1016/j.jocn.2019.06.019, indexed in Pubmed: 31221579.

26. Pail M, Brázdil M, Marecek R, et al. An optimized voxel-based morphometric study of gray matter changes in patients with left-sided and right-sided mesial temporal lobe epilepsy and hippocampal sclerosis (MTLE/HS). Epilepsia. 2010; 51(4): 511-518, doi: 10.1111/j.1528-1167.2009.02324.x, indexed in Pubmed: 19817822.

27. Besson P, Dinkelacker V, Valabregue R, et al. Structural connectivity differences in left and right temporal lobe epilepsy. Neuroimage. 2014; 100: 135-144, doi: 10.1016/j.neuroimage.2014.04.071, indexed in Pubmed: 24814212.

28. Coito A, Plomp G, Genetti M, et al. Dynamic directed interictal connectivity in left and right temporal lobe epilepsy. Epilepsia. 2015; 56(2): 207-217, doi: 10.1111/epi.12904, indexed in Pubmed: 25599821. 